ORIGINAL ARTICLE

\title{
Grading melanocytic dysplasia in paraffin wax embedded tissue by the nucleic acid index
}

\author{
D M Berman, S Wincovitch, S Garfield, M J Romeo
}

J Clin Pathol 2005;58:1206-1210. doi: 10.1136/icp.2005.026559

See end of article for authors' affiliations

Correspondence to: Dr D M Berman, BristolMyers Squibb, Princeton, NJ, USA; david.berman@ bms.com

Accepted for publication 11 March 2005
Background: Although nucleic acid derangements are the hallmark of melanocytic dysplasia, the gold standard for its diagnosis remains the microscopic evaluation of haematoxylin and eosin stained slides. However, light microscopy is subjective and crucial genomic changes do not always show as changes in histology.

Aims: To introduce the nucleic acid index (NAl) as a means of analysing nucleic acid derangements in histological sections at the level of the individual cell and within the context of its microenvironment. Methods: Confocal laser scanning microscopy was performed on melanocytic lesions stained with acridine orange (AO), a fluorescent stain for DNA and RNA. The NAI, calculated by measuring the fluorescence intensities of $A O$ in nuclei relative to the surrounding cytoplasm, reflects the concentration of DNA relative to RNA.

Results: When applied to benign naevi, dysplastic naevi, and melanoma, a very strong significant association was seen between lower NAl and malignant potential $(p<0.0001)$. Strong inverse correlations were found between NAl and both mitotic index and Breslow thickness. Interestingly, the NAI for dysplastic naevi is between that of melanoma and most benign naevi, consistent with their intermediate biological behaviour and histological appearance.

Conclusion: By providing a quantitative measure for melanocytic neoplasia, the NAl may improve the diagnosis of melanocytic lesions and the selection of treatment.
M elanoma, a highly aggressive disease that is increasing in incidence, is thought to arise via a series of mutations in melanocytes. ${ }^{12}$ The archetype for the development of this tumour is the familial atypical mole melanoma syndrome, wherein genetically unstable naevi (dysplastic melanocytic naevi; DMN) give rise to melanoma in situ, which then progresses to malignant melanoma. ${ }^{34}$ Transformation of melanocytes to a malignant phenotype may also occur in association with benign naevi, or even in the absence of evident precursor lesions. ${ }^{5}$

"It is crucial to diagnose melanoma accurately because early tumours can be cured by excision"

Melanocytic lesions may be difficult to classify and incorrect diagnoses are frequent causes of litigation. ${ }^{6}$ It is crucial to diagnose melanoma accurately because early tumours can be cured by excision. Diagnosing and grading DMN is also important because they are not only precursors to melanoma but are also markers for increased risk of melanoma. ${ }^{7}$ Although criteria for diagnosing dysplasia have been published, their application is notoriously subjective. ${ }^{8-10}$ For example, even among experts, there is only moderate concordance $(\kappa=0.5)$ in the grading of DMN. ${ }^{11}$ Unfortunately, adjunctive tests (for example, MIB-1 index and morphometry) for discriminating melanocytic dysplasia rely on light microscopy and are themselves also subjective. ${ }^{12}$

One promising alternative to light microscopy is confocal laser scanning microscopy (CLSM), which combines high resolution with optical sectioning and the ability to use multilabelled probes. Although primarily used in cell biology, CLSM has been selectively applied to paraffin wax embedded tissue. For example, CLSM of breast and prostate lesions can distinguish between benign and malignant nuclei. ${ }^{13}{ }^{14}$ However, these protocols are not practical for routine pathology practice. Furthermore, these analyses were not quantitative and thus do not greatly improve upon the current gold standard of haematoxylin and eosin (H\&E) microscopy. Unlike prostate and breast cancer, benign melanocytic lesions may exhibit subtle nuclear and architectural disorder, and there is even controversy as to whether nuclear changes alone can distinguish early dysplasia from benign precursors. ${ }^{9}$

Because abnormalities in nucleic acids are the cause of melanocytic dysplasia, genomic analysis of melanoma may aid in the diagnosis of difficult lesions. In fact, DNA has been studied extensively in melanoma tissue. ${ }^{15}$ However, genomic analysis requires extraction and pooling of a group of cells, thus losing information about individual cells and their histological distribution. In situ hybridisation, which can be performed in tissue sections, requires target specific probes and cannot assess global genetic changes. Technologies such as nuclear morphometry and argyrophilic staining of the nucleolar organising region have been used to study melanoma tissue; however, they are not amenable to routine use and provide only indirect evidence of DNA changes. ${ }^{12}{ }^{16-19}$

Cellular RNA concentrations positively correlate with tumour proliferative potential, ${ }^{20-23}$ but because of technical limitations, global RNA values have not been well studied in formalin fixed, paraffin wax embedded (FFPE) tumour tissue. The simultaneous quantitative analysis of both RNA and DNA at the level of the individual cell and within the context of its tissue microenvironment would be a powerful tool to study cancer. One potentially useful tool for such a study is the fluorescent nucleic acid dye acridine orange

Abbreviations: $\mathrm{AO}$, acridine orange; $\mathrm{CLSM}$, confocal laser scanning microscopy; DAPI, 4',6-diamidino-2-phenylindole; DMN, dysplastic melanocytic naevi; FFPE, formalin fixed, paraffin wax embedded; $\mathrm{H} \& \mathrm{E}_{\text {, }}$ haematoxylin and eosin; NAl, nucleic acid index; PBS, phosphate buffered saline; SR, surface rendering 
(AO), which has been used in benign FFPE tissues to label RNA and DNA. ${ }^{24-26}$

In this context, CLSM was used to discriminate 28 benign, atypical, and malignant FFPE melanocytic lesions, each stained with AO and 4',6-diamidino-2-phenylindole (DAPI). The objective was to develop a quantitative index to aid in the diagnosis and treatment selection of melanocytic lesions. Our data show that the fluorescence intensity of $\mathrm{AO}$ in the nucleus relative to the cytoplasm (nucleic acid index) can distinguish between benign and malignant melanocytic lesions.

\section{MATERIALS AND METHODS}

\section{Histological sections and slide preparation}

Histological FFPE sections ( $5 \mu \mathrm{m}$ thick) of benign and malignant melanocytic lesions were received as anonymised samples and with appropriate institutional ethical review board approval. H\&E stained slides were reviewed for each specimen. The benign lesions included nine intradermal naevi and one compound naevus. In addition to one metastatic melanoma, we evaluated 12 primary melanomas and one melanoma in situ from all cutaneous sites, with a mean Breslow thickness of $2.3 \mathrm{~mm}(0.34-7.6 \mathrm{~mm})$ and representing a wide range of cytologies and mitotic counts. Five dysplastic compound naevi were graded based on established criteria. $^{8-10}$

Unstained slides were dewaxed in xylene and rehydrated through descending grades of ethanol and eventually into water. The slides were incubated for five minutes in phosphate buffered saline (PBS) supplemented with $0.2 \%$ Triton X-100, washed with PBS, and then stained with $1 \mu \mathrm{g} /$ ml AO (Molecular Probes; Eugene, Oregon, USA) for 30 minutes in the dark. The slides were then washed three times with PBS for five minutes each, air dried, and coverslipped using DAPI containing mounting media (Molecular Probes).

\section{Confocal analysis}

The slides were examined with a Zeiss LSM 510 confocal microscope (Carl Zeiss Inc, Thornwood, New York, USA) with an Axiovert 100M inverted microscope. For each digital image $(512 \times 512$ pixels $)$, approximately 45 optical slices were collected at $0.2 \mu \mathrm{m}$ intervals using the Zeiss AIM software (v3.2) with a $100 \times 1.3$ NA Zeiss Plan-Neofluor oil immersion objective. Using a multitrack configuration, the AO and DAPI signals were collected with a BP 505-550 filter and with a BP 385-470 filter after excitation with a $488 \mathrm{~nm}$ and $364 \mathrm{~nm}$ laser line, respectively. All settings were kept constant except for the detector gain, which was adjusted to just under saturation, thereby eliminating saturated pixels. Volume rendering and surface rendering (SR) were performed on images using Imaris software v4.0.3 (Bitplane AG, Basil, Switzerland) with a $5 \mu \mathrm{m}$ grid. To determine the nucleic acid index, a representative $0.2 \mu \mathrm{m}$ slice was selected from 10 benign naevi, five dysplastic naevi, 11 melanomas, and one melanoma in situ. Average fluorescent intensities of $\mathrm{AO}$ were measured in nuclei and the surrounding cytoplasm using the Zeiss LSM software. Three to six nuclei and surrounding cytoplasms were measured with regions of
A

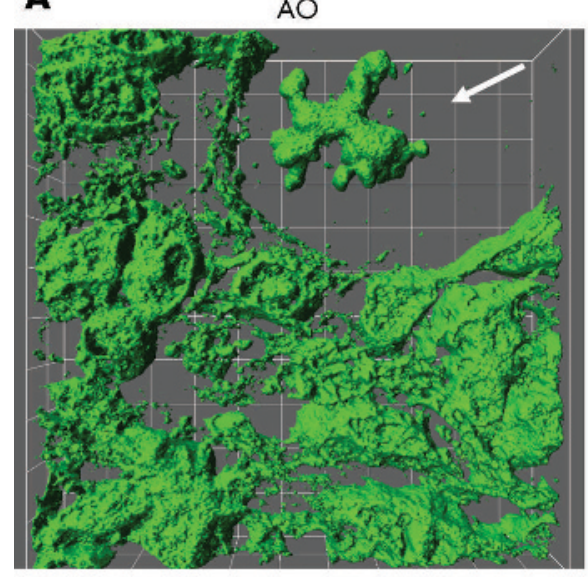

c

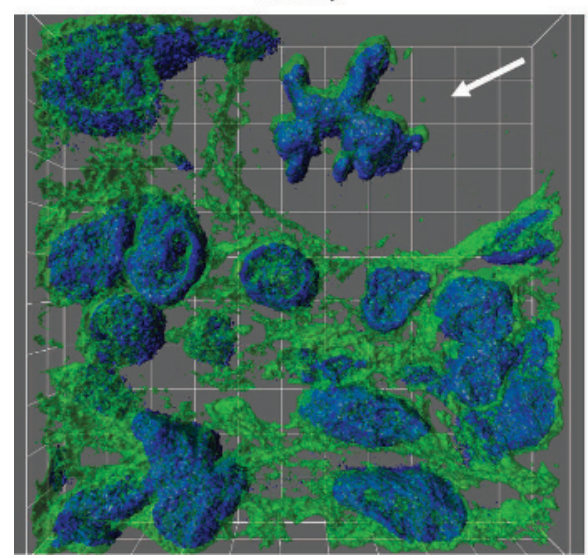

B

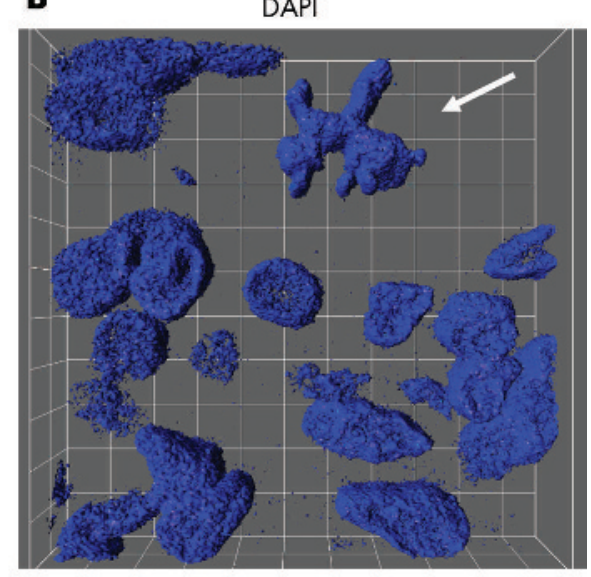

D

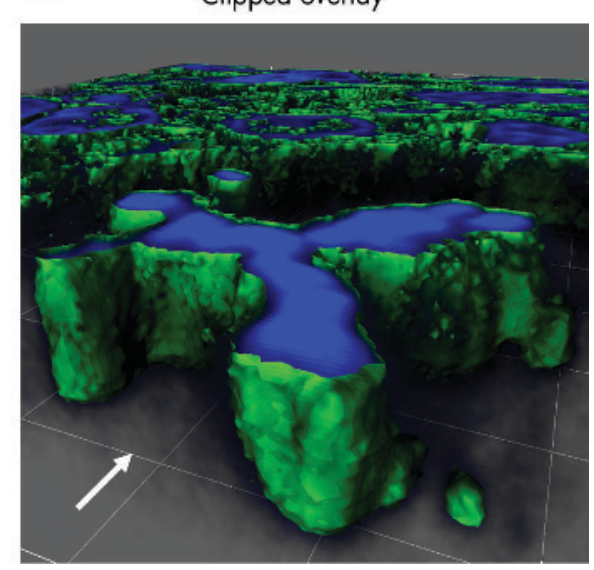

Figure 1 Three dimensional surface renderings of melanoma stained with (A) acridine orange (green) and (B) DAPI (blue). (C) The images have been overlayed. (D) The same image was clipped along the $Z$ axis to provide a side view of the tissue section. Note the mitotic figure (arrow). Images are projected on a $5 \mu \mathrm{m}$ grid. 
A
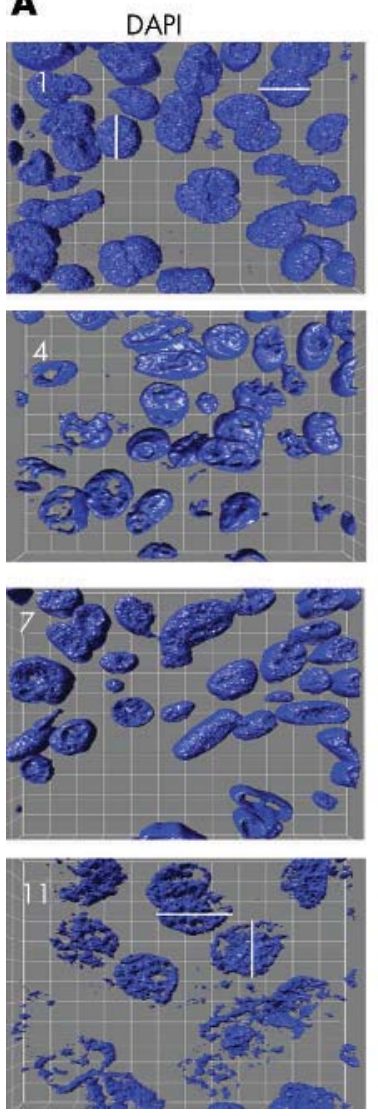
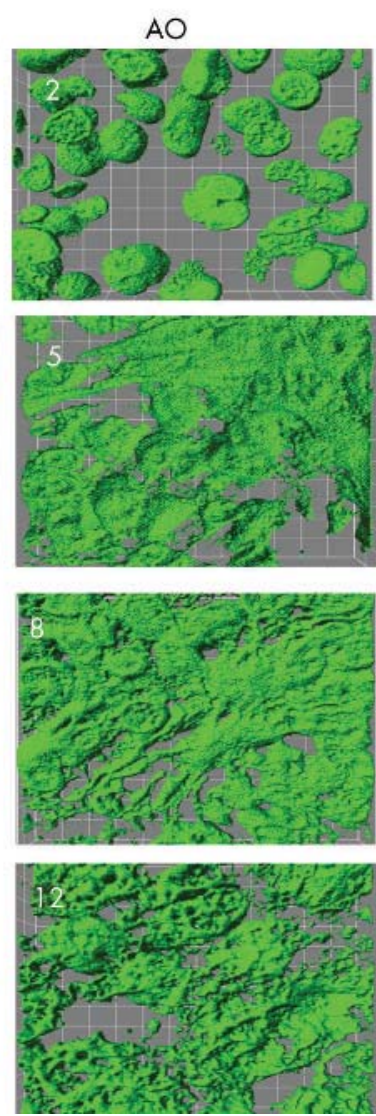
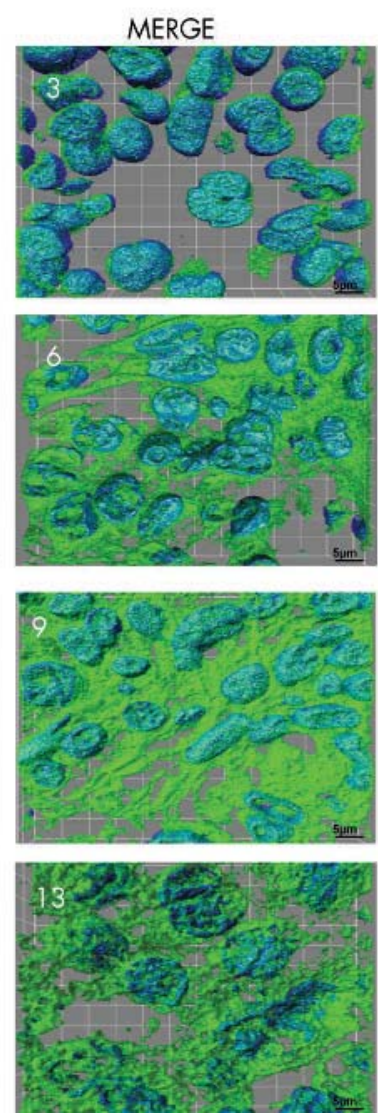

B

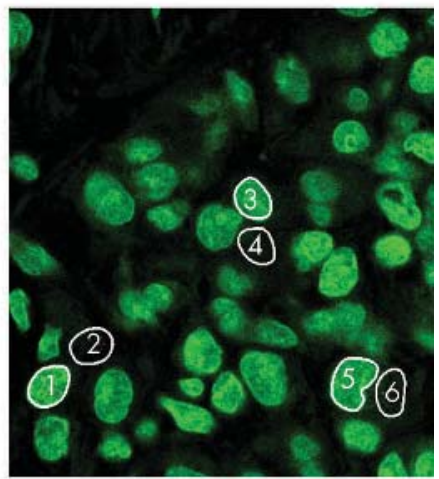

C

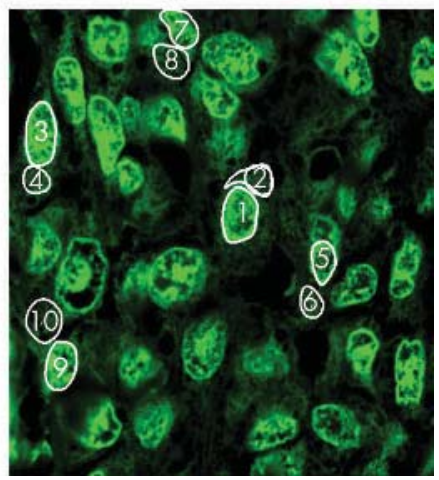

Figure 2 (A) Representative three dimensional surface renderings of benign naevi (1-3), dysplastic melanocytic naevi (4-6), melanoma in situ (7-8), and melanoma (9-12). Slides were stained with DAPI (blue) and acridine orange (green). A white bar through the equator of the DNA surface renderings (left panel) is included to illustrate the lack of symmetry in melanoma. (B, C) Measuring the nucleic acid index (NAl). The mean fluorescence intensities of acridine orange in a defined region of interest (white circles) of the nucleus (odd numbers) and surrounding cytoplasm (even numbers) were measured. The NAI was calculated by averaging the ratio of the nuclear to cytoplasmic intensities for three to six cells/slide. Representative optical sections of (B) benign naevus without detectable cytoplasm and (C) malignant melanoma.

interest of varying dimensions and shapes to determine the mean and standard deviation for each specimen.

\section{RESULTS}

FFPE tissue sections $(5 \mu \mathrm{m})$ were stained with two fluorescent nucleic acid dyes: AO and DAPI. DAPI, which binds to DNA, is concentrated in the nucleus. AO binds to RNA and DNA, thus staining the cytoplasm and the nucleus. Selective treatment with RNase and DNase showed that $90 \%$ of the nuclear staining with AO results from DNA, whereas cytoplasmic staining results from RNA. $^{25-27}$ The stained sections were optically sectioned at $0.2 \mu \mathrm{m}$ intervals by CLSM and composite three dimensional SRs of RNA and DNA were calculated (fig 1). Rotation of this image provides a unique three dimensional side profile of the tissue section (fig 1D).

Three dimensional SRs of nucleic acids were calculated for a wide range of melanocytic lesions and evaluated by visual inspection as a function of their $\mathrm{H} \& \mathrm{E}$ based diagnosis. Melanoma was characterised by an irregular DNA shell with a heterogeneous distribution of nuclear DNA, resulting in a "Swiss cheese" appearance (fig 1B, 2A). DNA SRs from benign naevi were more uniform and displayed bilateral symmetry around an imaginary line drawn through their central long axis (fig 2A). Cytoplasmic RNA SRs of melanoma were more expansive and hierarchically branched relative to benign naevi (fig 2A). In fact, cytoplasmic AO staining was not even identified in seven of the 10 benign naevi (table 1 ).
As expected, five DMN had nucleic acid distributions that were more asymmetrical than benign naevi but not as irregular as melanoma. One example of melanoma in situ resembled that of invasive melanoma. However, visual inspection of SR is still subjective and does not improve on the gold standard H\&E.

To develop a quantitative correlate for melanocytic atypia and malignant potential, the intensity of $\mathrm{AO}$ fluorescence was measured in representative $0.2 \mu \mathrm{m}$ optical sections from each lesion. Because measuring absolute nucleic acid values is not possible, a nucleic acid index (NAI) was calculated by measuring the fluorescence intensity of $\mathrm{AO}$ in the nucleus relative to the surrounding cytoplasm (fig $2 \mathrm{~B}$ ). As mentioned above, cytoplasmic AO correlates with RNA, whereas $90 \%$ of nuclear staining is the result of DNA. Therefore, the NAI closely parallels the ratio of DNA (nucleus) to RNA (cytoplasm). DAPI staining was used only to highlight the nuclear DNA and was not included in the quantitative analysis.

The NAI was analysed for one metastatic melanoma and 27 primary cutaneous melanocytic lesions, including benign naevi, dysplastic naevi, and melanoma (table 1). The mean NAI for melanoma was 2.0 (SD, 0.6). In contrast, cytoplasmic AO could not even be detected in seven of the benign naevi, precluding calculation of the NAI. In the remaining three naevi, the mean NAI was 4.4 (SD, 0.8). By H\&E, these last three naevi had no distinguishing histological features relative to the other seven naevi. The NAI for dysplastic 
Table 1 Nucleic acid index of melanocytic lesions

\begin{tabular}{|c|c|c|c|}
\hline Mean (SD) NAI & Diagnosis, site & Breslow thickness & Mitotic index (/ $10 \mathrm{HPF})$ \\
\hline $1.2(0.3)$ & Malignant melanoma, foot & 7.6 & 8 \\
\hline $1.4(0.1)$ & Malignant melanoma, back & 6 & 10 \\
\hline $1.5(0.2)$ & Malignant melanoma, eyebrow & 4.0 & $10 \S$ \\
\hline $1.6(0.5)$ & Malignant melanoma, back & 2.6 & 30 \\
\hline $1.6(0.1)$ & Metastatic melanoma, axilla & $N / D$ & N/D \\
\hline $1.7(0.1)$ & Malignant melanoma, knee & 7.0 & 5 \\
\hline $1.7(0.2)$ & Melanoma in situ, ear & $N / D$ & $N / D$ \\
\hline $2.2(0.3)$ & Malignant melanoma, ankle & 0.53 & 5 \\
\hline $2.2(0.4)$ & Malignant melanoma, head & 2.0 & 5 \\
\hline $2.7(0.2)$ & Malignant melanoma, back & 4.4 & 3 \\
\hline $2.7(0.7)$ & Malignant melanoma, chest & 1.87 & $5^{*}$ \\
\hline $2.7(0.3)$ & Malignant melanoma, breast & 0.34 & $5^{*}$ \\
\hline $2.8(0.6)$ & Malignant melanoma, back & 1.2 & 5 \\
\hline $2.9(0.5)$ & Dysplastic naevus, abdomen & $N / D$ & $N / D$ \\
\hline $3.5(0.4)$ & Benign naevus, head & $\mathrm{N} / \mathrm{D}$ & $\mathrm{N} / \mathrm{D}$ \\
\hline $3.6(0.5)$ & Dysplastic naevus, groin & N/D & N/D \\
\hline $4.2(0.3)$ & Dysplastic naevus, breast & $N / D$ & $N / D$ \\
\hline $4.3(0.5)$ & Benign naevus, neck & $N / D$ & $N / D$ \\
\hline $4.6(0.3)$ & Dysplastic naevus, not provided & $\mathrm{N} / \mathrm{D}$ & $\mathrm{N} / \mathrm{D}$ \\
\hline $5.1(0.5)$ & Benign naevus, axilla & $\mathrm{N} / \mathrm{D}$ & N/D \\
\hline $5.8(0.8)$ & Dysplastic naevus, back & $\mathrm{N} / \mathrm{D}$ & N/D \\
\hline$N / D$ & Seven benign naevi & $N / D$ & $N / D$ \\
\hline
\end{tabular}

naevi ranged from 2.9 to 5.8. The NAI value did not correlate with cell size, biopsy procedure, or body location (table 1 and data not shown).

Even excluding the seven benign samples with no detectable cytoplasmic staining, significant correlations were found between the NAI and the three categories of lesion: benign naevi, DMN, and melanoma. By an exact JonckheereTerpstra trend test $^{28}$ the two tailed $\mathrm{p}$ value for the comparison between NAI and the three groups (benign, dysplastic, and malignant), was $2.2 \times 10^{-5}$ (or p $<0.0001$ ), confirming a consistent trend between decreasing NAI and benign to dysplastic to malignant lesions. Thus, even based on available data (without the added benefit of the seven benign naevi), there was a highly significant association between NAI and benign, dysplastic, and malignant categories. By Spearman (non-parametric) correlation analysis of the 11 primary melanomas, strong inverse correlations were found between NAI and Breslow thickness $(-0.70$; $\mathrm{p}=0.016)$ and between NAI and mitotic index $(-0.76$; $\mathrm{p}=0.0062$ ).

\section{DISCUSSION}

Melanoma arises via dysplasia of cutaneous melanocytes, either in the context of a pre-existing naevus or de novo. The histology of a melanocytic lesion (for example, DMN) cannot always be correlated with its biological potential, and incorrect diagnoses are not infrequently rendered. Moreover, microscopic evaluation of $\mathrm{H} \& \mathrm{E}$ stained tissue sections is inherently subjective, contributing to frequent discordant diagnoses. The NAI was developed as a quantitative tool to improve the accuracy and diagnostic reproducibility of difficult melanocytic lesions.

\section{"Because the nucleic acid index does not rely on subjective evaluation, it should be superior to current adjunctive tests that rely on scoring by a pathologist"}

Because melanocytic dysplasia is driven by derangements in nucleic acids, we developed this technology to study nucleic acid abnormalities in FFPE tissue at the level of the individual cell. In fact, a highly significant association was found between lower NAI and malignant potential $(p<0.0001)$. Because the NAI does not rely on subjective evaluation, it should be superior to current adjunctive tests that rely on scoring by a pathologist.

The NAI is a quantitative estimate of the ratio of cellular DNA to RNA. Previous studies have shown that total RNA values are raised as a function of the tumour proliferation rate. ${ }^{20-23}$ Thus, the NAI provides a quantitative correlate for the proliferative potential of melanocytic lesions. Indeed, strong inverse correlations were found between NAI and both mitotic index and Breslow thickness. NAI may be helpful in the correct diagnosis of borderline lesions without identifiable mitotic bodies or when only limited tissue is available for study. A larger study is required to determine whether the NAI is an independent prognostic factor.

DMN represent a biologically and histologically intermediate stage of dysplasia. In addition to DMN being a precursor to melanoma, the grade of the melanocytic dysplasia correlates with the risk of developing melanoma. ${ }^{7}$ Because light microscopy is inherently subjective, there is a need for a quantitative assay to diagnose and grade these difficult lesions. In a small sample of DMN, the calculated NAI was intermediate between that of benign naevus and melanoma. Interestingly, whereas $70 \%$ of the benign naevi had no detectable cytoplasmic AO staining, three had NAI values in a similar range to dysplastic naevi. It is tempting to speculate that these three naevi may have a biological potential or predictive value similar to that of DMN. Alternatively, a low NAI may reflect the previous observations that a subset of benign naevi may harbour mutations in Ras or B-Raf. ${ }^{29} 30$

\section{Take home messages}

- We have developed a method, the nucleic acid index (NAI), that can quantitatively measure melanocytic neoplasia

- The NAl could provide an alternative/adjunct to light microscopy, which has the disadvantage of being subjective and that crucial genomic changes do not always show as changes in histology

- The NAl may improve the diagnosis of melanocytic lesions and the selection of treatment 
These results are intriguing and warrant additional study in an attempt to develop quantitative criteria that better reflect biological potential. A quantitative index will improve interobserver reliability and aid in clinical studies of DMN progression and risk of melanoma.

This protocol was designed with the practising pathologist in mind. Routine histological slides of $5 \mu \mathrm{m}$ thick sections of formalin fixed, paraffin wax embedded tissues were used. Slide preparation included a single 30 minute incubation step. This technology may easily be applied to study unstained slides from archives and community based practices.

NAI may reduce the interobserver variability inherent in evaluating pigmented lesions by microscopy. Apart from the requirement of a confocal laser scanning microscope and three dimensional SR software, the processing involved a simple 30 minute stain of archived FFPE $5 \mu \mathrm{m}$ tissue sections. We are currently analysing a series of atypical and unusual pigmented lesions to develop objective criteria that may provide clinically relevant information for those lesions that are not easily classified by H\&E.

\section{ACKNOWLEDGEMENTS}

The authors acknowledge the expert assistance of Dr S Steinberg (NCI) for help with the statistics.

\section{Authors' affiliations}

D M Berman, M J Romeo, Laboratory of Pathology, National Cancer Institute, Bldg 10-2N212, 10 Center Drive Bethesda, MD 20896, USA S Wincovitch, S Garfield, Laboratory of Experimental Carcinogenesis, National Cancer Institute, Bethesda, MD 20892, USA

$S$ Wincovitch and S Garfield contributed equally to this work.

\section{REFERENCE}

1 Lens MB, Dawes M. Global perspectives of contemporary epidemiological trends of cutaneous malignant melanoma. Br J Dermatol 2004;150:179-85

2 Schaffer JV, Rigel DS, Kopf AW, et al. Cutaneous melanoma-past, present, and future. J Am Acad Dermatol 2004;51(suppl 1):S65-9

3 Clark WH Jr, Reimer RR, Greene M, et al. Origin of familial malignant melanomas from heritable melanocytic lesions. "The B-K mole syndrome". Arch Dermatol 1978;114:732-8.

4 Frichot BC 3rd, Lynch HT, Guirgis HA, et al. ew cutaneous phenotype in familial malignant melanoma. Lancet 1977;1:864-5.

5 Gruber SB, Barnhill RL, Stenn KS, et al. Nevomelanocytic proliferations in association with cutaneous malignant melanoma: a multivariate analysis. J Am Acad Dermatol 1989;21:773-80.

6 Xu X, Elder DE. A practical approach to selected problematic melanocytic lesions. Am J Clin Pathol 2004;121(suppl):S3-32.

7 Arumi-Uria M, McNutt NS, Finnerty B. Grading of atypia in nevi: correlation with melanoma risk. Mod Pathol 2003;16:764-71.

8 Elder D. Melanocytic tumors of the skin. Washington DC: Armed Forces Institute of Pathology, 1990.
9 Barnhill R. Pathology of melanocytic nevi and malignant melanoma. New York: Springer-Verlag, 2004

10 Crowson AN, Magro CM, Mihm MC. The melanocytic proliferations, 1st ed. New York: Wiley-Liss, 2001.

11 Farmer ER, Gonin R, Hanna MP. Discordance in the histopathologic diagnosis of melanoma and melanocytic nevi between expert pathologists. Hum Pathol 1996;27:528-31.

12 Li LX, Crotty KA, Scolyer RA, et al. Use of multiple cytometric markers improves discrimination between benign and malignant melanocytic lesions: a study of DNA microdensitometry, karyometry, argyrophilic staining of nucleolar organizer regions and MIB1-Ki67 immunoreactivity. Melanoma Res 2003;13:581-6.

13 Liu S, Weaver DL, Taatjes DJ. Three-dimensional reconstruction by confocal laser scanning microscopy in routine pathologic specimens of benign and malignant lesions of the human breast. Histochem Cell Biol 1997; 107:267-78.

14 Irinopoulou T, Vassy J, Beil M, et al. Three-dimensional DNA image cytometry by confocal scanning laser microscopy in thick tissue blocks of prostatic lesions. Cytometry 1997;27:99-105.

15 Bastian BC. Understanding the progression of melanocytic neoplasia using genomic analysis: from fields to cancer. Oncogene 2003;22:3081-6

16 Bruijn JA, Berwick M, Mihm MC Jr, et al. Common acquired melanocytic nevi, dysplastic melanocytic nevi and malignant melanomas: an image analysis cytometric study. J Cutan Pathol 1993;20:121-5.

17 Li LX, Crotty KA, Palmer AA, et al. Argyrophilic staining of nucleolar organizer region count and morphometry in benign and malignant melanocytic lesions. Am J Dermatopathol 2003;25:190-7.

18 Pilch H, Gunzel S, Schaffer U, et al. Evaluation of DNA ploidy and degree of DNA abnormality in benign and malignant melanocytic lesions of the skin using video imaging. Cancer 2000;88:1370-7.

19 Wiltgen M, Gerger A, Wagner C, et al. Discrimination of benign common nevi from malignant melanoma lesions by use of features based on spectral properties of the wavelet transform. Anal Quant Cytol Histol 2003;25:243-53

20 Hiraki $M$. Intracellular DNA and RNA in the course of tumor cell growth. Kurume Med J 1994;41:1-13.

21 El-Naggar AK, Kemp BL, Sneige N, et al. Bivariate RNA and DNA content analysis in breast carcinoma: biological significance of RNA content. Clin Cancer Res 1996;2:419-26.

22 Pollack A, Prudhomme DL, Greenstein DB, et al. Flow cytometric analysis of RNA content in different cell populations using pyronin $Y$ and methyl green. Cytometry 1982;3:28-35

23 Traganos F, Darzynkiewicz Z, Melamed MR. The ratio of RNA to total nucleic acid content as a quantitative measure of unbalanced cell growth. Cytometry 1982;2:212-18.

24 Ginsberg SD, Galvin JE, Chiu TS, et al. RNA sequestration to pathological lesions of neurodegenerative diseases. Acta Neuropathol (Berl) 1998;96:487-94.

25 Dutt MK. Acridine orange-its use in the specific staining of DNA in mammalian tissue sections. Microsc Acta 1981;84:37-42.

26 Sarnat HB, Seagram GF, Trevenen CL, et al. A fluorochromic stain for nucleic acids to demonstrate submucosal and myenteric neurons in Hirschsprung's disease. Am J Clin Pathol 1985;83:722-5.

27 Gill JE, Wheeless LL Jr, Hanna-Madden C, et al. A comparison of acridine orange and Feulgen cytochemistry of human tumor cell nuclei. Cancer Res 1978;38:1893-8.

28 Hollander M, Wolfe D. Non-parametric statistical methods, 2nd ed. New York: John Wiley and Sons, 1999.

29 Pollock PM, Harper UL, Hansen KS, et al. High frequency of BRAF mutations in nevi. Nat Genet 2003;33:19-20.

30 Kumar R, Angelini S, Snellman E, et al. BRAF mutations are common somatic events in melanocytic nevi. J Invest Dermatol 2004;122:342-8. 